\title{
Rapid detection of multidrug-resistant Mycobacterium tuberculosis using the mycobacteria growth indicator tube (MGIT) system
}

M.A.S. Telles, A. Bori,

A.B.R. Amorim, A.F. Cruz, M.I.T. Pini and D.N. Sato
Instituto Adolfo Lutz, São Paulo, SP, Brasil

\section{Correspondence \\ M.A.S. Telles \\ Setor de Micobactérias \\ Instituto Adolfo Lutz \\ Av. Dr. Arnaldo, 355, 90 andar \\ 01246-902 São Paulo, SP \\ Brasil \\ Fax: +55-11-3085-3505 \\ E-mail: atelles@ial.sp.gov.br \\ M.A.S. Telles is a member of RELACTB (Tuberculosis Network for Latin America and the Caribbean) that receives support from the European Commission RDG (INCO-DEV Programme) (project No. ICA4-CT- 2001-10087).}

Publication supported by FAPESP. ....................

Received July 31, 2001

Accepted June 20, 2002

\begin{abstract}
The emergence of multidrug-resistant strains of Mycobacterium tuberculosis has increased the need for rapid drug susceptibility tests, which are needed for adequate patient treatment. The objective of the present study was to evaluate the mycobacteria growth indicator tube (MGIT) system to detect multidrug-resistant $M$. tuberculosis strains. The MGIT system was compared with two standard methods (proportion and resistance ratio methods). One hundred clinical M. tuberculosis isolates [25 susceptible to isoniazid (INH) and rifampicin (RIF), 20 resistant to INH, 30 resistant to INH-RIF, and 25 resistant to INH-RIF and other drugs] obtained in the State of São Paulo were tested for INH and RIF susceptibility. Full agreement among the tests was found for all sensitive and all INH-resistant strains. For RIF-resistant strains results among the tests agreed for 53 (96.4\%) of 55 isolates. Results were obtained within 6 days (range, 5 to 8 days), 28 days and 12 days when using MGIT, the proportion method and the resistance ratio methods, respectively. The MGIT system presented an overall agreement of $96 \%$ when compared with two standard methods. These data show that the MGIT system is rapid, sensitive and efficient for the early detection of multidrug-resistant $M$. tuberculosis.
\end{abstract}

\section{Introduction}

Tuberculosis has once again become a reason for concern in almost all parts of the world and especially in developing countries. Due to the shortage of laboratory resources, the diagnosis of tuberculosis in these countries is based on microscopic examination. This practice does not permit the isolation of the agent and the determination of the infectious strain's susceptibility profile. Therefore, the treatment usually begins in the absence of information concerning drug susceptibility (1).

About $95 \%$ of the Mycobacterium tuberculosis isolates obtained from new cases of tuberculosis without previous treatment are susceptible to standard antituberculosis drugs. For these cases, treatment with first-line drugs usually leads to cure (2). However, in the presence of drug resistance, susceptibility tests must be done as soon as possible to allow the physician to control the dissemination of multidrug-resistant $M$. tuberculosis. 
Therefore, the emergence of multidrugresistant tuberculosis has increased the need for rapid drug susceptibility tests. The methods currently available use solid media (proportion, resistance ratio, and absolute concentration) and the radiometric BACTEC 460TB system (Becton-Dickinson Microbiology System, Sparks, MD, USA) (3-5). Methods that use solid media take 3 to 4 weeks to produce conclusive results, while the BACTEC 460TB system, which was the first method based on a liquid medium, provides results more rapidly. The system, however, is radiometric and due to its radioactive nature requires special equipment and radioactivity safety measures.

More recently, a new mycobacteria growth indicator tube (MGIT, Becton-Dickinson) was developed as an alternative, nonradiometric method for the detection of mycobacteria using a fluorescent oxygenquenched sensor embedded in silicone at the bottom of tubes (6-10). The MGIT system is read manually, requiring only a UV lamp, and the tubes are easily inoculated. Preliminary studies of drug susceptibility testing of M. tuberculosis strains have shown promising results (11-13).

We have evaluated the use of the MGIT system specifically to detect strains of $M$. tuberculosis resistant to isoniazid (INH) and rifampicin (RIF) in 100 clinical isolates obtained in the State of São Paulo.

\section{Material and Methods}

\section{Microorganisms}

One hundred M. tuberculosis clinical isolates with previously known resistance profiles were used: 25 INH- and RIF-susceptible, 20 INH-resistant, and 55 multidrugresistant strains (30 of them INH-RIF resistant and 25 resistant to INH-RIF and other drugs). The strain was considered to be multidrug resistant when it was resistant to INH and RIF.

\section{Inoculum preparation}

Several colonies from a 3-week-old Lowenstein-Jensen (LJ) slant were subcultured in $4.0 \mathrm{ml}$ of $7 \mathrm{H} 9$ broth, and incubated at $37^{\circ} \mathrm{C}$ for 10 days. After this period of time $2.0 \mathrm{ml}$ of this culture was transferred to a tube and the turbidity adjusted with $7 \mathrm{H} 9$ broth to a No. 1 McFarland standard. This suspension was further diluted 1:5 with sterile distilled water.

\section{MGIT susceptibility testing}

Susceptibility testing was performed according to the protocol provided by the manufacturer. The final concentrations of each antibiotic in the test tubes were $0.1 \mathrm{mg} / \mathrm{ml}$ INH and $1.0 \mathrm{mg} / \mathrm{ml} \mathrm{RIF.} \mathrm{The} \mathrm{reading} \mathrm{of} \mathrm{the}$ test was started at day 3 after inoculation, using a BACTEC MicroMGIT Reader (Becton-Dickinson). The growth control tube was compared to the positive and negative controls. The day when the growth control tube gave a positive result was considered day 0 for the purpose of interpretation of the drugcontaining tubes; if the growth control tubes remained negative, the reading was continued until day 12 after inoculation. On the day the growth control tube became positive, the drug-containing tubes were read and interpreted according to manufacturer recommendations. A strain was considered to be susceptible if the drug-containing tube did not fluoresce within two days of the date of the positivity of the growth control, and resistant if the drug-containing tube was positive within 2 days of the date of the positivity of the growth control.

\section{Proportion and resistance ratio methods}

All the strains were also tested by two gold standard methods, i.e., the proportion and resistance ratio methods in $\mathrm{LJ}$ medium, based on standard procedures $(14,15)$. The drug concentrations used were $0.2 \mu \mathrm{g} / \mathrm{ml}$ 
INH and $40.0 \mu \mathrm{g} / \mathrm{ml}$ RIF for the proportion method, and 0.5 to $0.2 \mu \mathrm{g} / \mathrm{ml} \mathrm{INH}$ and 2.5 to $10 \mu \mathrm{g} / \mathrm{ml} \mathrm{RIF}$ for the resistance ratio method. We considered the strains to be resistant or sensitive to INH and RIF without considering resistance to other drugs that were not tested in the present study.

\section{Results and Discussion}

The MGIT method detected INH- and RIF-resistant strains, despite their different resistance patterns. Total agreement was found for the sensitive strains between the two standard methods and MGIT (Table 1). Among the 20 strains resistant only to INH, the MGIT method showed complete agreement, whereas there was disagreement between the two standard methods: one strain was found to be resistant to INH and RIF by the resistance ratio method and two strains were resistant to INH and RIF by the proportion method. Among the 55 strains resistant to INH and RIF there was disagreement between the standard methods and MGIT for two strains, which were sensitive to RIF by MGIT. Another strain resistant to both INH and RIF by the method originally used to identify it, was sensitive to INH by the resistance ratio method.

Table 2 shows a comparison of the results of the MGIT versus standard methods for INH-sensitive and INH-resistant strains and Table 3 shows a similar comparison of the results of the MGIT versus standard methods for RIF-sensitive and RIF-resistant strains. Whereas there was complete agreement between the tests with regard to INH sensitivity, discrepant results were obtained for two strains with regard to RIF sensitivity. Two strains identified as RIF resistant by both standard methods were sensitive to RIF by the MGIT method (Table 3 ).

Therefore, there was a $100 \%$ correlation between MGIT and standard methods concerning resistance to INH, while for RIF there was a $98 \%$ correlation. In a similar
Table 1. Susceptibility of Mycobacterium tuberculosis strains to isoniazid (INH) and rifampicin (RIF) tested by the mycobacteria growth indicator tube (MGIT) and the proportion and resistance ratio methods.

\begin{tabular}{lcccc}
\hline Resistance pattem & \multicolumn{4}{c}{ Number of strains } \\
\cline { 2 - 5 } & Previous test & Resistance ratio & Proportion & MGIT \\
\hline INH + RIF sensitive & 25 & 25 & 25 & 25 \\
INH resistant & 20 & 19 & 18 & 20 \\
INH + RIF resistant & 55 & 54 & 55 & 53
\end{tabular}

Table 2 - Comparison of results of the mycobacteria growth indicator tube (MGIT) versus standard methods for isoniazid (INH).

\begin{tabular}{lcc}
\hline MGIT & \multicolumn{2}{c}{ Standard methods } \\
\cline { 2 - 3 } & INH resistant & INH sensitive \\
\hline INH resistant & 75 & 0 \\
INH sensitive & 0 & 25
\end{tabular}

Sensitivity: $75 / 75=100 \%$; specificity: $25 / 25=$ $100 \%$; positive predictive value: $75 / 75=100 \%$; negative predictive value: $25 / 25=100 \%$; accuracy: $100 / 100=100 \%$

Table 3. Comparison of results of the mycobacteria growth indicator tube (MGIT) versus standard methods for rifampicin (RIF).

\begin{tabular}{lcc}
\hline MGIT & \multicolumn{2}{c}{ Standard methods } \\
\cline { 2 - 3 } & RIF resistant & RIF sensitive \\
\hline RIF resistant & 54 & 0 \\
RIF sensitive & 2 & 44 \\
\hline
\end{tabular}

Sensitivity: 54/56 = 96.4\%; specificity: $44 / 44=$ $100 \%$; positive predictive value: $54 / 54=100 \%$; negative predictive value: $44 / 46=95.6 \%$; accuracy: $98 / 100=98 \%$.

study by Palomino et al. (16), 100\% agreement was found regarding INH, $98 \%$ for RIF, 99\% for ethambutol and 91\% for streptomycin. Similar results have been reported in other studies $(11,17)$.

MGIT susceptibility results are usually obtained within 8 days (range: 5 to 13 days) $(11,16,17)$. This speed is the main advantage 
of the test when compared with standard methods that use solid medium, which are routinely used in developing countries.

The recommended treatment for tuberculosis includes a combination of RIF and $\mathrm{INH}$; therefore, M. tuberculosis strains resistant to both of these drugs are designated multidrug resistant. The emergence of multidrug-resistant tuberculosis represents a major threat to the control of tuberculosis, and it has been shown that the major cause of multidrug resistance is the use of poor tuberculosis control strategies (18). As part of a tuberculosis control program, it is very important to monitor drug sensitivity patterns in the community and individual patients with chronic tuberculosis after treatment failure. Understanding drug resistance patterns in a community is also of great epidemio- logical significance since it provides indicators of the existence and prevalence of primary and acquired drug resistance, essential to evaluate the quality of the tuberculosis control program (19).

The results of the present study indicate that a possible way to overcome the problem of the scarce resources of public health laboratories in developing countries like Brazil would be to adopt the MGIT system as a screening test for multidrug-resistant $M$. $t u$ berculosis strains, thus allowing for rapid identification of patients who need special treatment and isolation conditions.

\section{Acknowledgments}

We thank Becton-Dickinson for providing the BBL MGIT tubes.

\section{References}

1. Enarson DA, Rieder $\mathrm{HL} \&$ Arnadottir $\mathrm{T}$ (1994). Tuberculosis Guide for Low Income Countries. 3rd edn. International Union Against Tuberculosis and Lung Diseases, Paris, France.

2. Hawkins JE, Wallace RJ \& Brown BA (1991). Antibacterial susceptibility tests: mycobacteria. In: Balows A, Hausler WJ, Hermann KL, Isenberg HD \& Shadomy HJ (Editors), Manual of Clinical Microbiology. American Society for Microbiology, Washington, DC, USA, 1138-1152.

3. Kent PT \& Kubica GP (1985). Mycobacteriology: A Guide for the Level III Laboratory. US Department of Health and Human Services, Atlanta, GA, USA.

4. Canetti G, Fox W, Khomenko A, Mahler HT, Menon NK, Mitchison DA, Rist N \& Smelev NA (1969). Advances in techniques of testing mycobacterial drug sensitivity and the use of sensitivity test in tuberculosis control programmes. Bulletin of the World Health Organization, 41: 21-43.

5. Roberts GD, Goodman NL, Heifets L, Larsh HW, Lindner TH, McClatchy J K, McGinnis MR, Siddiqi $\mathrm{SH} \&$ Wright $\mathrm{P}$ (1983). Evaluation of the BACTEC radiometric method for recovery of mycobacteria and drug susceptibility testing of My- cobacterium tuberculosis from acid-fast smear positive specimens. J ournal of Clinical Microbiology, 18: 689-696.

6. Badak FZ, Kiska DI, Setterquist S, Hartley C, O'Connell MA \& Hopfer RI (1996). Comparison of mycobacteria growth indicator tube with BACTEC 460 for detection and recovery of mycobacteria from clinical specimens. J oumal of Clinical Microbiology, 34: 2236-2239.

7. Pfyffer GE, Welscher HM, Kissling $P$, Cieslak C, Casal MJ , Gutierrez J \& RuschGerdes S (1997). Comparison of the mycobacteria growth indicator tube (MGIT) with radiometric and solid culture for recovery of acid-fast bacilli. J ournal of Clinical Microbiology, 35: 364-368.

8. Sharp SE, Suarez CA, Lemes M \& Poppiti J r RJ (1996). Evaluation of the mycobacteria growth indicator tube compared to Septi-Chek AFB for the detection of mycobacteria. Diagnostic Microbiology and Infectious Disease, 25: 71-75.

9. Rivera $A B$, Tupasi $T E$, Grimaldo ER, Cardano RC \& Co VM (1997). Rapid and improved recovery of Mycobacterium tuberculosis in mycobacteria growth indicator tube combined with solid Lowenstein J ensen medium. International J oumal of Tuberculosis and Lung Disease, 1: 454-459.
10. Casal M, Gutierrez J \& Vaquero M (1997) Comparative evaluation of the mycobacteria growth indicator tube with the BACTEC 460 TB system and LowensteinJ ensen medium for isolation of mycobacteria from clinical specimens. International J oumal of Tuberculosis and Lung Disease, 1: 81-84.

11. Palaci M, Ueki SYM, Sato DN, Telles MAS, Curcio M \& Silva EAM (1996). Evaluation of mycobacteria growth indicator tube for recovery and drug susceptibility testing of Mycobacterium tuberculosis isolates from respiratory specimens. J ournal of Clinical Microbiology, 34: 762-764.

12. Reisner BS, Gatson AM \& Woods GL (1995). Evaluation of mycobacteria growth indicator tube for susceptibility testing of Mycobacterium tuberculosis to isoniazid and rifampin. Diagnostic Microbiology and Infectious Disease, 22: 325-329.

13. Walters SB \& Hanna BA (1996). Testing of susceptibility of Mycobacterium tuberculosis to isoniazid and rifampin by mycobacteria growth indicator tube method. J ournal of Clinical Microbiology, 34: 1565-1567.

14. Ministério da Saúde, Fundação Nacional da Saúde (1994). Manual de Bacteriologia da Tuberculose. 2nd edn. Centro de Referência Professor Hélio Fraga, Rio de 
J aneiro, RJ , Brazil.

15. Collins $\mathrm{CH}$, Grange J M \& Yates MD (1997). Tuberculosis Bacteriology. Organization and Practice. 2nd edn. Butterworth Heinemann, London, England.

16. Palomino J C, Traore $\mathrm{H}$, Fissette $\mathrm{K} \&$ Portaels F (1999). Evaluation of mycobacteria growth indicator tube (MGIT) for drug susceptibility testing of Mycobacterium tuberculosis. International J ournal of Tu- berculosis and Lung Disease, 3: 344-348.

17. Rüsch-Gerdes S, Domehl C, Nardi G, Gismondo MR, Welscher HM \& Pfyffer GE (1999). Multicenter evaluation of the mycobacteria growth indicator tube for testing susceptibility of Mycobacterium tuberculosis to first-line drugs. J oumal of Clinical Microbiology, 37: 45-48.

18. Chaulet P, Boulahal F \& Grosset J (1995). Surveillance of drug resistance for tuber- culosis control: why and how. Tubercle and Lung Disease, 76: 487-492.

19. World Health Organization Tuberculosis Programme and International Union Against Tuberculosis and Lung Disease (1994). Guidelines for Surveillance of Drug Resistance in Tuberculosis. WHO/TB/ 94.179. World Health Organization, Geneva, Switzerland. 